\section{PREVALÊNCIA E FATORES ASSOCIADOS À INCONTINÊNCIA URINÁRIA EM MULHERES IDOSAS}

\author{
Prevalence and factors associated with urinary incontinence \\ in elderly women
}

\author{
Prevalencia y factores asociados a la incontinencia urinaria \\ de mujeres mayores
}

Artigo Original

\section{RESUMO}

Objetivo: Verificar a prevalência da queixa de Incontinência Urinária (IU) e os fatores associados em idosas no município de Petrolina/PE. Métodos: Estudo transversal e exploratório realizado em 2009 com 172 idosas nas quais se avaliaram características sociodemográficas, socioeconômicas e estilo de vida. Para investigar a IU utilizou-se o International Consultation on Incontinence Questionnaire - Short Form" (ICIQ-SF) acrescido de questões obstétricas e de comorbidades. Dados trabalhados com intervalo de confiança a $95 \%$ utilizando o STATA ${ }^{\circledR}$ 9.0. Resultados: A queixa de IU esteve presente em $81(47,1 \%)$ idosas. A faixa etária maior que 75 anos de idade estava associada à IU (RP 1,57 IC95\% 1,57-11,09). Na associação com ocupação notou-se que tanto as idosas aposentadas (RP 4,64 IC95\% 0,98-21,98) quanto as donas de casa (RP 6,25 IC95\% 1,14-34,12) tinham maior ocorrência de referir IU, mas apenas a associação com a condição dona de casa foi significante. Quanto ao diabetes, idosas que tinham a doença (RP 1,57 IC95\% 1,16-2,13) tinham maior frequência de referir queixa de IU quando comparadas às idosas sem diabetes. Conclusão: Encontrou-se uma elevada prevalência de Incontinência Urinária em idosas, correspondendo a quase metade das mulheres investigadas, estando associada às idosas de maior idade, donas de casa e diabéticas.

Descritores: Incontinência Urinária; Saúde da Mulher; Envelhecimento.

\begin{abstract}
Objective: To verify the prevalence of urinary incontinence (UI) complaints and associated factors among elderly women in the municipality of Petrolina / PE. Methods: Exploratory cross-sectional study conducted in 2009 with 172 elderly women who were assessed regarding sociodemographic, socioeconomic and lifestyle characteristics. UI was assessed using The International Consultation on Incontinence Questionnaire - Short Form (ICIQ$S F)$ in addition to questions on obstetrics and comorbidities. Data were computed with a 95\% confidence interval using STATA 9.0. Results: The urinary incontinence was present in $81(47.1 \%)$ elderly women. The age group older than 75 years was associated with UI (PR 1.57 CI 95\% 1.57 - 11.09). Regarding the association with occupation, it was verified that both the retired elders (PR 4.64 CI 95\% 0.98 - 21.98) and housewives (PR 6.25 CI 95\% 1.14 - 34.12) were more likely to report UI, but only the association with the housewife condition was significant. With regard to diabetes, elderly women who had the disease (PR 1.57 CI $95 \% 1.16$ - 2.13) were more likely to report urinary incontinence when compared to elderly women without diabetes. Conclusion: There was a high prevalence of urinary incontinence among elderly women, which accounted for almost half of the women assessed and was associated with older age, housewives and diabetes.
\end{abstract}

Descriptors: Urinary Incontinence; Women's Health; Aging.

\author{
Karla Veruska Marques \\ Cavalcante $^{(1)}$ \\ Maria Isabel Gomes da Cruz \\ Silva $a^{(2)}$ \\ Adan Smith Ferreira Bernardo ${ }^{(2)}$ \\ Damião Ernane Souza ${ }^{(3)}$ \\ Thereza Christina da Gama \\ Cunha Lima ${ }^{(4)}$ \\ Adriana Gomes Magalhães ${ }^{(2)}$
}

1) Universidade Federal da Paraíba - UFPB - João Pessoa - Brasil

2) Universidade Federal do Rio Grande do Norte - UFRN - Trairi (RN) - Brasil

3) Instituto Brasileiro de Geografia e Estatística - IBGE/ UE/RN - Natal (RN) -

Brasil

4) Universidade de Pernambuco - UPE Petrolina (PE) - Brasil

Recebido em: 19/06/2013 Revisado em: 27/08/2013 Aceito em: 16/09/2013 


\section{RESUMEN}

Objetivo: Verificar la prevalencia de queja de Incontinencia Urinaria (IU) y los factores asociados en mujeres mayores del municipio de Petrolina/PE. Métodos: Estudio transversal y exploratorio realizado en 2009 con 172 mujeres mayores en las cuales se evaluaron las características socio demográficas, socio económicas y el estilo de vida. Para investigar la IU se utilizó del International Consultation on Incontinence Questionnaire - Short Form" (ICIQ-SF) y otras preguntas sobre obstetricia y comorbilidades. Los datos fueron evaluados con intervalo de confianza del $95 \%$ utilizando el STATA ${ }^{\circledR}$ 9.0. Resultados: La queja de IU se presentó en 81 (47,1\%) mujeres mayores. La franja de edad de mujeres mayores de 75 años estuvo asociada a la IU (RP 1,57 IC95\% 1,57-11,09). Al asociar la IU y la ocupación se notó que las mujeres mayores jubiladas (RP 4,64 IC95\% 0,98-21,98) y las amas de casa (RP 6,25 IC95\% 1,14-34,12) refirieron más IU, sin embargo, solamente la asociación de la condición de ama de casa fue significativa. Respecto a la diabetes, las mujeres mayores que tenian la enfermedad (RP 1,57 IC95\% 1,16-2,13) presentaron mayor frecuencia de queja de IU al comparar con aquellas sin diabetes. Conclusión: Se encontró una elevada prevalencia de $I U$ en mujeres mayores que corresponde casi a la mitad de las mujeres investigadas con asociación entre las que tienen más edad, las amas de casa y diabéticas.

Descriptores: Incontinencia Urinaria; Salud de la Mujer; Envejecimiento.

\section{INTRODUÇÃO}

O controle da micção evolui de um mecanismo reflexo na primeira infância para o controle voluntário na vida adulta através de processos neurofisiológicos, volitivos e reflexos, que controlam o esvaziamento vesical e o controle esfincteriano $^{(1)}$. O trato urinário inferior apresenta alterações relacionadas ao envelhecimento, em ambos os gêneros, contudo, como fenômeno isolado, não é causa deste transtorno, apesar de induzir às modificações funcionais e estruturais no sistema urinário as quais predispõem ao problema $^{(2)}$. Entretanto, determinadas condições, como alterações histológicas, traumas e diversas co-morbidades, podem afetar o controle da micção ${ }^{(3)}$.

ASociedade Internacional de Continência (International Continence Society - ICS) definiu a Incontinência Urinária (IU) como "perda involuntária de urina"(4). Embora a IU não faça parte do envelhecimento fisiológico, observase um aumento de sua prevalência entre pessoas idosas ${ }^{(5)}$. A IU é uma condição multifatorial e um problema de saúde significante na sociedade moderna, atingindo, no mundo, mais de 50 milhões de pessoas, principalmente as mulheres $^{(5)}$, numa relação de ocorrência de 2-5 mulheres para 1 homem $^{(6)}$.
Embora as taxas de prevalência da IU variem de acordo com a definição e características dos estudos e população alvo ${ }^{(7)}$, é estimado que $39 \%$ da população feminina adulta com mais de 60 anos apresentem sintomas de incontinência ${ }^{(6,8,9)}$. No Brasil, são poucos os estudos atuais sobre a prevalência de incontinência urinária, essa estimativa varia entre $20 \%$ e $35 \%$ para mulheres entre 50 e $75 \operatorname{anos}^{(10)}$.

Segundo a etiologia e fisiopatologia, a IU pode ser classificada como IU de esforço (IUE), urgeincontinência (IU) ou IU mista (IUM) e tem determinação multifatorial, podendo comprometer a fase de enchimento e/ou esvaziamento vesical, ou ainda ser desencadeada por doenças neuromusculares, fragilidade do sistema de suporte, gravidez, alterações hormonais, câncer, sintomas de apresentação de outras doenças, entretanto, o papel de cada uma dessas condições e a real causa da IU ainda é algo que demanda investigação ${ }^{(11)}$.

Estima-se que o governo dos Estados Unidos gaste em torno de 10-16 bilhões de dólares com mulheres incontinentes por ano ${ }^{(12,13)}$. No Brasil, não há estimativas quanto aos gastos anuais. Contudo, estudos apontam que a IU pode ter um impacto negativo na qualidade de vida das pessoas com essa queixa ${ }^{(12,14)}$ interferindo diretamente nas atividades diárias dos idosos, de tal maneira que um ciclo vicioso de ansiedade e sofrimento, além de um sentimento de vergonha, os leva a um importante incômodo psicológico e variado grau de isolamento social ${ }^{(14)}$. E, como condição que afeta o estado geral da saúde dos sujeitos acometidos, é possível que a IU possa afetar também a saúde autorreferida (SAR), que é uma medida do estado de saúde frequentemente utilizada e que apresenta forte capacidade preditora para morbi-mortalidade ${ }^{(15-17)}$. A SAR incorpora múltiplos aspectos da saúde física e social dos sujeitos e tem apresentado associação significante com vários desfechos relacionados ao envelhecimento ${ }^{(18)}$, como a IU.

Apesar da importância da IU, as mulheres relatam uma variedade de motivos que as levam a não procurar atenção médica, como a desvalorização dos sintomas, como se a perda urinária fosse uma ocorrência natural do processo de envelhecimento ${ }^{(13)}$, o que implica na falta de conhecimento da real dimensão da problemática.

Neste sentido, considerando que a percepção negativa de saúde seja um preditor de busca por serviços de saúde, que incontinência urinária é um problema de saúde negligenciado e cercado de preconceito, o objetivo desse estudo foi verificar a prevalência da queixa de Incontinência Urinária (IU) e os fatores associados em idosas no município de Petrolina/PE. Assim, o estudo poderá contribuir para a compreensão do fenômeno e consequentemente auxiliar na orientação ou reorientação das políticas públicas de saúde, 
com vistas à promoção da saúde e qualidade de vida dessa parcela da população.

\section{MÉTODOS}

Trata-se de estudo transversal, de base comunitária com caráter exploratório, realizado no período de setembro a dezembro de 2009.

Avaliaram-se 172 idosas com idade igual ou superior a 60 anos, adstritas às Unidades de Saúde da Família, participantes de grupos comunitários de idosas e moradoras de comunidades em bairros do município de Petrolina- PE e que não apresentavam déficit de cognição, sendo estes os critérios de inclusão no estudo. Já os critérios de exclusão compreendiam idosas que apresentassem déficit cognitivo.

Por meio de um sorteio na lista do Cadastro Nacional de Estabelecimentos de Saúde (CNES/DATASUS), definiramse as Unidades de Saúde da Família participantes do presente estudo, em um total de 04 unidades, equivalentes a $10 \%$ das unidades do município. Para as comunidades, foram sorteados 04 bairros, correspondentes também a $10 \%$ dos bairros do município, e em cada um destes, se sortearam 02 setores censitários a partir dos arquivos de mapas e setores do Instituto Brasileiro de Geografia e Estatística (IBGE). Entretanto, os dois centros de convivência de idosos foram escolhidos por conveniência, devido à escassez de registros nos órgãos competentes sobre a quantidade e a localização deste tipo de organização no município.

Nas Unidades de Saúde e nos Centros de Idosos, realizou-se a definição das entrevistadas a partir de sorteio em cada dia de visita do estudo. Nos bairros, realizou-se trajeto anti-horário, com coleta de dados em domicílio alternado, e nos casos de não existência de idosa no domicílio sorteado, domicílio fechado ou recusa em responder ao questionário, este era aplicado na residência imediatamente posterior que apresentasse idosa com 60 anos ou mais.

Para a coleta, aplicou-se um questionário para investigação de características sociodemográficas (idade, estado civil e escolaridade), características socioeconômicas (renda e trabalho) e sobre o estilo de vida (consumo de álcool, fumo e prática de atividade física). E para investigação especifica da IU utilizou-se o questionário "International Consultation on Incontinence Questionnaire - Short Form" (ICIQ-SF) traduzido e validado para a língua portuguesa ${ }^{(19)}$. Cada item desses domínios teve sua resposta aferida em uma escala do tipo Likert de cinco pontos. Neste instrumento, acrescentaram-se perguntas sobre o tipo de parto e o tipo de assistência realizado durante o parto, bem como, uma questão que investigava a presença de diabetes.

Definiu-se a presença de incontinência urinária (IU) através do critério no qual a idosa era considerada incontinente quando respondesse que perdia urina pelo menos uma vez por semana, mesmo que em pequena quantidade, e que essa perda interferia ou não em sua vida ${ }^{(3)}$.

Com relação às características reprodutivas e a morbidade autorreferida, a variável filhos foi agrupada em duas categorias, sim e não, respectivamente, a respeito da presença ou ausência de filhos; a quantidade de filhos, agrupada em três categorias, a saber, nenhum, de 1 a 5 filhos e mais de 5 filhos; o tipo de parto categorizado em três estratos, não teve filhos, parto natural e parto cesariano; e quanto ao tipo de assistência prestada no parto utilizaramse as categorias, não teve filhos, hospital público, hospital privado, parteira ou parto em domicílio. Quanto à morbidade referida, a presença de diabetes foi classificada em sim e não de acordo com a presença; da mesma forma que a variável incontinência urinária.

Os dados foram analisados utilizando o pacote estatístico STATA ${ }^{\circledR}$ 9.0, através do qual se calcularam frequências absolutas e relativas das variáveis categóricas, e a média e o desvio padrão das contínuas. Calculou-se também a prevalência, a razão de prevalência (RP) e o respectivo intervalo de confiança a 95\% (IC 95\%).

Os procedimentos de abordagem, coleta e análise de dados obedeceram a Resolução $n^{0}$ 196/96 do Conselho Nacional de Saúde, tendo sido aprovado pelo Comitê de Ética e Pesquisa - CEP/UPE da Universidade de Pernambuco através do parecer $n^{\circ} 124 / 2009$.

\section{RESULTADOS}

Das 172 idosas avaliadas do município de Petrolina/ $\mathrm{PE}$, a maioria $(\mathrm{n}=90 ; 52,4 \%)$ tinha entre 65 e 75 anos de idade, $109(63,4 \%)$ era separada/viúva/divorciada e tinha de 1 a 4 anos de escola $(n=74 ; 43,1 \%)$. Quanto à renda, a maior parte recebia mensalmente até 1 salário mínimo $(\mathrm{n}=$ $144 ; 83,7 \%$ ) e tinha como principal ocupação a condição de aposentada $(\mathrm{n}=133 ; 77,4 \%)$. Das 172 idosas, $125(72,7 \%)$ eram chefe de família. Quanto aos hábitos de vida, a maioria das idosas não praticava atividade física $119(69,1 \%)$, não consumia bebida alcoólica $168(97,7 \%)$ e não fumava 148 $(86,1 \%)$ (Tabela I).

Em relação às características reprodutivas, 156 idosas $(90,7 \%)$ tinham filhos, das quais $118(75,2 \%)$ tinham mais de 4 filhos. A maioria teve parto natural $(\mathrm{n}=147$; 93,6\%) com auxílio de parteira ou no próprio domicílio $(\mathrm{n}=89$; $56,7 \%$ ). Fato interessante está relacionado à morbidade autorreferida entre as idosas, 45 referiam ter diabetes, apontando uma prevalência de 26,2\%. Enquanto que para incontinência urinária observou-se uma prevalência mais elevada, $47,1 \%(\mathrm{n}=81)$ das mulheres referiam apresentar a condição, conforme pode ser observado na Tabela II. 
Tabela I - Características sociodemográficas, socioeconômicas e de hábitos de vida em idosas. PetrolinaPE, 2009.

\begin{tabular}{|c|c|c|}
\hline Variáveis & $\mathbf{n}$ & $\%$ \\
\hline \multicolumn{3}{|l|}{ Idade } \\
\hline$\leq 65$ anos & 48 & 27,9 \\
\hline 66 a 75 anos & 90 & 52,4 \\
\hline$>75$ anos & 34 & 19,7 \\
\hline \multicolumn{3}{|l|}{ Estado Civil } \\
\hline Solteira & 17 & 9,9 \\
\hline Casada ou União Estável & 46 & 26,7 \\
\hline Separada/viúva/divorciada & 109 & 63,4 \\
\hline \multicolumn{3}{|l|}{ Anos de escola } \\
\hline Nenhum & 73 & 42,4 \\
\hline De 1 a 4 anos & 74 & 43,1 \\
\hline Cinco anos ou mais & 25 & 14,5 \\
\hline \multicolumn{3}{|l|}{ Renda (em salários mínimos) } \\
\hline$\leq 1$ & 144 & 83,7 \\
\hline$>1$ & 28 & 16,3 \\
\hline \multicolumn{3}{|l|}{ Ocupação } \\
\hline Trabalho Formal & 12 & 6,9 \\
\hline Aposentada & 133 & 77,4 \\
\hline Dona de casa & 27 & 15,7 \\
\hline \multicolumn{3}{|l|}{ Posição na família } \\
\hline Chefe de família & 125 & 72,7 \\
\hline Membro da família & 47 & 27,3 \\
\hline \multicolumn{3}{|l|}{ Atividade física semanal } \\
\hline Não pratica & 119 & 69,1 \\
\hline Até três vezes & 40 & 23,3 \\
\hline Mais de três vezes & 13 & 7,6 \\
\hline \multicolumn{3}{|l|}{ Consumo de álcool } \\
\hline Não & 168 & 97,7 \\
\hline Sim & 4 & 2,3 \\
\hline \multicolumn{3}{|l|}{ Consumo de fumo } \\
\hline Não & 148 & 86,1 \\
\hline Sim & 24 & 13,9 \\
\hline
\end{tabular}

A Tabela III apresenta a análise dos fatores associadas à incontinência urinária (IU). A faixa etária maior que 75 anos de idade estava associada à IU (RP 1,57; IC95\% 1,57-11,09). Quando comparado ao estado civil solteiro, as idosas do estado civil casado ou união estável tinham menor frequência de ter queixa de IU (RP 0,79; IC95\% $0,26-2,42)$, enquanto as idosas do estado civil separado/ viúvo/divorciado tinham maior probabilidade de apresentar IU (RP 1,10; IC95\% 0,39-3,07), e observaram-se diferenças significativas.
Em relação à escolaridade, idosas que tinha de 1 a 4 anos de escolaridade e aquelas com cinco anos ou mais tinham maior probabilidade de referir queixa de IU (RP 1,28; IC95\% 0,67-2,45 e RP 1,18; IC95\% 0,472,94 , respectivamente), mas a associação não apresentou significância para ambos os níveis de escolaridade. Para a renda não se observou associação significante com IU (RP 1,07; IC95\% 0,71-1,62) (Tabela III).

Tabela II - Características reprodutivas e de morbidade autorreferida em idosas. Petrolina-PE, 2009.

\begin{tabular}{lcc}
\hline Variáveis & $\mathbf{n}$ & $\mathbf{\%}$ \\
\hline Têm filhos & & \\
$\quad$ Sim & 156 & 90,7 \\
$\quad$ Não & 16 & 9,3 \\
Número de filhos & & \\
$\quad$ De 1 a 4 filhos & 39 & 24,8 \\
$\quad$ > filhos & 118 & 75,2 \\
Tipo de parto & & \\
$\quad$ Natural & 147 & 93,6 \\
$\quad$ Cesariano & 10 & 6,4 \\
Tipo de assistência & & \\
$\quad$ Hospital público & 66 & 42,0 \\
$\quad$ Hospital privado & 2 & 1,3 \\
$\quad$ Parteira ou em domicílio & 89 & 56,7 \\
Diabetes autorreferida & & \\
$\quad$ Sim & 45 & 26,2 \\
$\quad$ Não & 127 & 73,8 \\
Queixa de incontinência urinária & & \\
$\quad$ Sim & 81 & 47,1 \\
$\quad$ Não & 91 & 52,9 \\
\hline
\end{tabular}

Quando avaliada a associação com ocupação, observouse que tanto as idosas aposentadas (RP 4,64; IC95\% 0,9821,98 ) quanto as donas de casa (RP 6,25; IC95\% 1,1434,12 ) tinham maior frequência em referir IU, mas apenas a associação com donas de casa tinha significância estatística. $\mathrm{Na}$ avaliação do papel da posição na família na queixa de IU, não se observou diferença estatisticamente significante em ser chefe de família ou apenas membro com a possibilidade em referir IU (RP 1,26; IC95\% 0,91-1,71) (Tabela III).

Em relação à atividade física semanal, idosas que praticavam até três vezes por semana (RP 0,59 IC95\% 0,28$1,23)$ e idosas que praticavam mais de três vezes por semana (RP 0,84 IC95\% 0,27-2,66), tinham menor probabilidade de apresentar queixa de IU quando comparadas as idosas não praticantes de atividade física, mas a associação não demonstrou significância estatística. O hábito de fumar também não apresentou associação significante com a queixa de IU (RP 0,97; IC95\% 0,61-1,54). 
Tabela III - Prevalência e razão de prevalência bruta (IC95\%) da incontinência urinária de acordo com as covariáveis de interesse em idosas. Petrolina/PE, 2009

\begin{tabular}{|c|c|c|c|c|}
\hline Variáveis & $\mathbf{n}$ & Prevalência da IU & RP & IC $(95 \%)$ \\
\hline \multicolumn{5}{|l|}{ Idade } \\
\hline$\leq 65$ anos & 48 & 43,7 & 1,00 & - \\
\hline 66 a 75 anos & 90 & 37,8 & 0,78 & $(0,38-1,59)$ \\
\hline$>75$ anos & 34 & 76,5 & 4,18 & $(1,57-11,09)$ \\
\hline \multicolumn{5}{|l|}{ Estado Civil } \\
\hline Solteira & 17 & 47,1 & 1,00 & - \\
\hline Casada ou União estável & 46 & 41,3 & 0,79 & $(0,26-2,42)$ \\
\hline Separada/ Viúva/Divorciada & 109 & 49,5 & 1,10 & $(0,39-3,07)$ \\
\hline \multicolumn{5}{|l|}{ Anos de escola } \\
\hline Nenhum & 73 & 43,8 & 1,00 & - \\
\hline De 1 a 4 anos & 74 & 50,0 & 1,28 & $(0,67-2,45)$ \\
\hline Cinco anos ou mais & 25 & 48,0 & 1,18 & $(0,47-2,94)$ \\
\hline \multicolumn{5}{|l|}{ Renda (em SM) } \\
\hline$\leq 1$ & 144 & 46,5 & 1,00 & - \\
\hline$>1$ & 28 & 50,0 & 1,07 & $(0,71-1,62)$ \\
\hline \multicolumn{5}{|l|}{ Ocupação } \\
\hline Trabalho formal & 12 & 16,7 & 1,00 & - \\
\hline Aposentada & 133 & 48,1 & 4,64 & $(0,98-21,98)$ \\
\hline Dona de casa & 27 & 55,6 & 6,25 & $(1,14-34,12)$ \\
\hline \multicolumn{5}{|l|}{ Posição na família } \\
\hline Chefe de família & 125 & 44,0 & 1,00 & - \\
\hline Membro da família & 47 & 55,3 & 1,26 & $(0,91-1,74)$ \\
\hline \multicolumn{5}{|l|}{ Atividade física semanal } \\
\hline Não pratica & 119 & 50,4 & 1,00 & - \\
\hline Até três vezes & 40 & 37,5 & 0,59 & $(0,28-1,23)$ \\
\hline Mais de três vezes & 13 & 46,1 & 0,84 & $(0,27-2,66)$ \\
\hline \multicolumn{5}{|l|}{ Consumo de fumo } \\
\hline Não & 148 & 47,3 & 1,00 & - \\
\hline Sim & 24 & 45,8 & 0,97 & $(0,61-1,54)$ \\
\hline \multicolumn{5}{|l|}{ Ter filhos } \\
\hline Não & 156 & 37,5 & 1,00 & - \\
\hline Sim & 16 & 48,1 & 1,28 & $(0,67-2,46)$ \\
\hline \multicolumn{5}{|l|}{ Número de filhos } \\
\hline De 1 a 4 filhos & 39 & 35,9 & 1,00 & - \\
\hline Mais de 4 filhos & 118 & 52,5 & 1,46 & $(0,93-2,30)$ \\
\hline \multicolumn{5}{|l|}{ Tipo de parto } \\
\hline Normal & 147 & 51,0 & 1,00 & - \\
\hline Cesariano & 10 & 60,0 & 0,82 & $(0,37-1,77)$ \\
\hline \multicolumn{5}{|l|}{ Tipo de assistência } \\
\hline Hospital público & 66 & 46,9 & 1,00 & - \\
\hline Hospital privado & 2 & 50,0 & 1,13 & $(0,07-18,8)$ \\
\hline Parteira ou em domicílio & 89 & 49,4 & 1,10 & $(0,58-2,09)$ \\
\hline \multicolumn{5}{|l|}{ Diabetes (autorreferida) } \\
\hline Não & 127 & 40,9 & 1,00 & - \\
\hline Sim & 45 & 64,4 & 1,57 & $(1,16-2,13)$ \\
\hline
\end{tabular}

IU=Incontinência Urinária; $\mathrm{n}=$ número da amostra; $\mathrm{RP}=$ razão de prevalência; $\mathrm{IC}=$ intervalo de confiança; $\mathrm{SM}=$ salário mínimo. 
$\mathrm{Na}$ avaliação das características reprodutivas, observou-se que idosas que não têm filhos apresentam maior probabilidade de referir queixa de IU com RP 1,18 (IC95\% 0,67-2,46), semelhante às idosas que relataram ter tido mais de 4 filhos com RP 1,46 (IC95\% 0,93-2,30), porém não foi observado diferença estatística. Ainda sobre as características reprodutivas, quando comparadas às idosas que relataram o parto normal, as idosas submetidas ao parto cesariano tinham menor probabilidade de referir queixa de IU (OR 0,8; IC 95\% 0,37-1,77), mas as diferenças não foram estatisticamente significantes.

Por fim, outro dado relevante foi a associação significativa entre diabetes autorreferida e a queixa de IU, ou seja, idosas que referiam ter diabetes tinham maior probabilidade de referir queixa de IU quando comparadas as idosas sem a doença com RP 1,57 (IC95\% 1,16-2,13).

\section{DISCUSSÃO}

No presente estudo identificou-se elevada prevalência de IU em idosas (47,1\%), cujo perfil majoritário foi de mulheres que tinha idade superior a 65 anos, viviam sem parceiro (separada/viúva/divorciada), tinham baixa escolaridade, baixa renda, possuindo como principal rendimento a aposentadoria de até um salário mínimo, mas exercendo a função de chefe de família. Eram mulheres sedentárias, porém não consumiam bebida alcoólica e não fumavam. Dentre os fatores de risco avaliados, o dado relevante foi a associação estatisticamente significativa entre diabetes autorreferida e a queixa de IU.

Baseado nos estudos científicos desenvolvidos em todo o mundo, a IU tem uma elevada prevalência ${ }^{(10)}$, principalmente entre mulheres ${ }^{(9,20)}$, porém identifica-se uma larga variação entre os valores percentuais nos diferentes estudos $^{(8,9,10,20)}$. As divergências metodológicas, o tipo e o intervalo entre os estudos, são fatores a serem considerados que poderiam explicar a grande variação encontrada, com registros de $74(9) \%$ a $39 \%$ da população feminina adulta com mais de $60 \operatorname{anos}^{(6,8)}$. O presente estudo corrobora com os achados da literatura, apresentando uma prevalência de $47,1 \%(\mathrm{n}=81)$ para mulheres com queixa de IU, ou seja, quase a metade das idosas estudadas apresentaram perdas urinárias, o que remete a uma prevalência elevada de IU, como retrata a literatura.

Evidências científicas apontam para um perfil socioeconômico desfavorável de mulheres idosas ${ }^{(9,20)}$, como demonstra o estudo da zona urbana de Pouso Alegre/ MG, para a população em geral, encontrou prevalência de 65,9\% para mulheres (40-59 anos), com baixa escolaridade (84\%), elevado índice de analfabetismo ou apenas ensino fundamental $(61,8 \%)$ e que desenvolviam atividade/trabalho não remunerado $(50,7 \%)^{(9)}$. De maneira semelhante, um estudo $^{(20)}$ desenvolvido em Campinas/SP que também identificou elevada prevalência entre idosos, do gênero feminino, com queixa de IU (74\%), com idade superior a 70 anos (78\%), sem companheiros (59\%), morando com familiares $(75 \%)$, aposentados $(71 \%)$ e que apresentavam baixa escolaridade $(49 \%)^{(20)}$.

O presente estudo, desenvolvido em Petrolina/ PE, também identificou condições socioeconômicas desfavoráveis no tocante ao estado civil, escolaridade e renda mensal. Cerca de $63,4 \%$ das idosas encontravamse separadas/viúvas/divorciadas, $43,1 \%$ tinham baixa escolaridade com apenas 1 a 4 anos de escola e $83,7 \%$ baixa renda, recebia mensalmente até 1 salário mínimo.

Situações desvantajosas, como as mencionadas anteriormente, podem agravar determinadas condições de saúde como a IU, devido ao reduzido acesso a bens e serviços que podem implicar em menor envolvimento em programas de educação em saúde, reduzindo a busca por atendimento especializado ou por acreditar que a perda urinária é uma condição normal ao processo de envelhecimento ${ }^{(21)}$.

No atual estudo, a ocupação principal que apresentou associação significante entre idosas foi naquelas que exerciam apenas a função de dona de casa, com maiores chances de referir queixas de IU. Adicionalmente, demonstrou-se que a maioria das mulheres eram sedentárias $(69,1 \%)$. Porém, a prática de atividade física até 3 vezes por semana ou mais de três vezes por semana pelas idosas (OR 0,84 IC95\% 0,27-2,66), parecia diminuir as chances das mulheres apresentarem queixa de IU quando comparadas às idosas sedentárias. No entanto, a associação dos resultados não foi significativa.

Curiosamente, em uma pesquisa com programas de atividade física, apesar de estimularem relacionamentos sociais e promoverem uma melhor Qualidade de Vida, grande parte das idosas investigadas apresentou uma percepção de saúde negativa( ${ }^{(21)}$. Somado a estes dados, um estudo de revisão sobre a prática de atividade física e IU aponta que as mulheres que fazem atividade física, principalmente as mais vigorosas, apresentam maior probabilidade de apresentar perda involuntária de urina e este é motivo comum de afastamento das mulheres da prática de exercícios, entretanto, a revisão também aponta que se utilize como alternativas o uso de barreira física (absorvente) e redução da ingesta de líquidos, como estratégia para manutenção da prática de exercícios ${ }^{(22)}$.

Quanto ao hábito de fumar, 14\% das idosas tabagistas não demonstraram associação significante com a queixa de IU na atual investigação, apesar da literatura reportar que a prática do fumo e o etilismo estão relacionados a distúrbios do assoalho pélvico e a fatores de risco relevantes para a $\mathrm{IU}^{(23)}$.

As características obstétricas como número de filhos e tipo de parto não estavam significantemente associadas 
a queixas de IU no presente estudo, apesar de $75,2 \%$ das entrevistadas referirem que tinha tido mais de quatro filhos. Estes resultados divergem de outros estudos ${ }^{(24,25)}$ que apontam que tanto o parto vaginal como o número de gestações estão associados à IU. É possível que esses resultados não tenham sido encontrados no presente estudo em virtude de $56,7 \%$ das idosas ter referido que realizou o parto em domicílio ou com ajuda da parteira, contexto onde é incomum o uso de procedimentos como episiotomia e fórcipes. $\mathrm{O}$ uso do parto com fórcipes está relacionado com fator associado a $\mathrm{IU}^{(25)}$ e a episotomia rotineira como fator que afeta negativamente a força dos músculos do assoalho pélvico e pode determinar a $\mathrm{IU}^{(26)}$.

Idosas com diabetes apresentaram mais chance de referir queixa de IU quando comparadas às idosas sem diabetes na investigação em questão. Esses resultados estão de acordo com outro achado ${ }^{(27)}$ que observou que mulheres com diabetes referiam mais frequentemente a condição de IU quando comparadas às não diabéticas. Além disso, as mulheres com diabetes raramente referenciavam essa condição ao médico e acreditavam que a IU é uma condição $\operatorname{rara}^{(27)}$. Aspectos como alterações microvasculares que afetam o complexo assoalho pélvico-vesical, o aumento da frequência urinária devido à hiperglicemia, associadas às mudanças nos mecanismos neurofisiológicos do sistema genitourinário podem contribuir para maior queixa de IU entre pessoas com diabetes ${ }^{(28)}$.

No presente estudo, observou-se que a chance de referir IU aumentava com a idade. Na população geral, as várias alterações decorrentes do envelhecimento e a maior prevalência de comorbidades são importantes fatores associados à $\mathrm{IU}^{(3)}$. Entretanto, há uma maior proporção de mulheres com IU quando comparadas aos homens, sendo que a prevalência da IU em mulheres é quase cinco vezes maior do que em homens ${ }^{(9)}$. Esse fenômeno pode ser explicado pelo fato de que as alterações orgânicas ocorridas na senescência podem ser potencializadas por características típicas ao gênero feminino, como a história reprodutiva que expõe o trato genitourinário a maiores traumas e pelas alterações hormonais decorrentes do climatério $^{(29)}$.

Os resultados encontrados na presente pesquisa devem ser avaliados com parcimônia, pois as associações encontradas apresentaram um largo intervalo de confiança que podem estar relacionadas com a medida utilizada para definir a IU. Além disso, se trata de um estudo de corte transversal que impossibilita maiores conclusões sobre os fenômenos estudados, bem como, a não realização de testes mais específicos para o diagnóstico ou a confirmação da IU, sendo essa condição baseada apenas na queixa das idosas entrevistadas. Entretanto, o presente estudo aponta para a necessidade de novos estudos que associem a avaliação clínica com exames específicos para avaliar a função e disfunção do trato urinário como o urodinâmico ${ }^{(30,}$
${ }^{31)}$, com o propósito de aclarar aspectos obscuros sobre os determinantes da IU.

\section{CONCLUSÃO}

Encontrou-se uma elevada prevalência de Incontinência Urinária em idosas, correspondendo a quase metade das mulheres investigadas, estando associada às idosas de maior idade, donas de casa e diabéticas. No entanto, estudos adicionais, com uma maior abrangência, fazem-se necessários para melhor retratar a realidade das mulheres idosas no município de Petrolina/PE.

\section{REFERÊNCIAS}

1. Yoshimura N, Miyazato M. Neurophysiology and therapeutic receptor targets for stress urinary incontinence. Int J Urol. 2012; 19(6):524-37.

2. Du Moulin MF, Hamers JPH, Ambergen AW, Halfens RJG. Urinary incontinence in older adults receiving home care diagnosis and strategies. Scand J Caring Sci. 2009; 23(2):222-30.

3. Reis RB, Cologna AJ, Martins ACP, Paschoalin EL, Tucci Jr S, Suaid HJ. Incontinência urinária no idoso. Acta Cir Bras. 2003;18(5):47-51.

4. Abrams P, Andersson KE, Birder L, Brubaker L, Cardozo L, Chapple $\mathrm{C}$, et al. Fourth International Consultation on Incontinence Recommendations of the International Scientific Committee: evaluation and treatment of urinary incontinence, pelvic organ prolapsed, and fecal incontinence. Neurourol Urodyn. 2010;29(1):213-40.

5. Buckley BS, Lapitan MCM. Epidemiology Committee of the Fourth International Consultation on Incontinence, Paris, 2008. Prevalence of urinary incontinence in men, women, and children - current evidence: findings of the Fourth International Consultation on Incontinence. Urology. 2010;76(2):265-70.

6. Sacomori C, Negri NB, Cardoso FL. Incontinência urinária em mulheres que buscam exame preventivo de câncer de colo uterino: fatores sociodemográficos e comportamentais. Cad Saúde Pública. 2013;29(6): $1251-9$.

7. DuBeau CE, Simon SE, Morris JN. The effect of urinary incontinence on quality of life in older nursing home residents. J Am Geriatr Soc. 2006;54(9):132533.

8. Caldas CP, Conceição IRS, José RMC, Silva BMC. Terapia comportamental para incontinência urinária da mulher idosa: uma ação do enfermeiro. Texto \& Contexto Enferm. 2010;19(4):783-8. 
9. Santos CRS, Santos VLCG. Prevalência da incontinência urinária em amostra randomizada da população urbana de Pouso Alegre, Minas Gerais, Brasil. Rev Latinoam Enferm. 2010;18(5):903-10.

10. Torrealba FCM, Oliveira LDR. Incontinência urinária na população feminina de idosas. Ensaios e Ciência. 2010;14(1):159-75.

11. Dedicação AC, Haddad M, Saldanha MES, Driusso P. Comparison of quality of life for different types of female incontinence urinary. Rev Bras Fisioter 2009; 13(2):116-22.

12. Frick AC, Huang AJ, Van den Eeden SK, Knight SK, Creasmna JM, Yang J, et al. Mixed urinary incontinence: greater impact on quality of life. J Urol 2009; 182(2):596-600.

13. Bushnell DM, Martin ML, Summers KH, Svihra J, Lionis C, Patrick DL. Quality of life of women with urinary incontinence: cross-cultural performance of 15 language versions of the I-QOL. Qual Life Res. 2005;14(8):1901-13.

14. Pedro AF, Ribeiro J, Soler ZASG, Bugdan AP. Qualidade de vida de mulheres com incontinência urinária. SMAD Rev Eletrônica Saúde Mental Álcool Drog. 2011;7(2):63-70.

15. Babones SJ. The consistency of self-rated health in comparative perspective. Public Health. 2009;123(2):199-201.

16. Idler EL, Benyamini Y. Self-rated health and mortality: a review of twenty-seven community studies. J Health Soc Behav. 1997;38(1):21-37.

17. Fayers PM, Sprangers MAG. Understanding self-rated health. Lancet. 2002; 359(9302):187-8.00

18. Damián J, Pastor-Barriuso R, Valderrama-Gama E. Factors associated with self-rated health in older people living in instituitions. BMC Geriatric.2008;8:5.

19. Tamanini JTN, Dambros M, D'Ancona CAL, Palma PCR, Rodrigues Netto Jr N. Validation of the "International Consultation on Incontinence Questionnaire - Short Form" (ICIQ-SF) for Portuguese. Rev Saúde Pública. 2004;38(3):438-44.

20. Silva VAD, D'Elboux MJ. Factores associeted with urinary incontinence in a Elderly individuals who meet frailty criteria. Texto \& Contexto Enferm. 2012;21(2):338-47.

21. Vagetti GC, Moreira NB, Barbosa Filho VC, Oliveira V, Cancian CF, Mazzardo O, Campos W. Domínios da qualidade de vida associados à percepção de saúde: um estudo com idosas de um programa de atividade física em bairros de baixa renda de Curitiba/PR. Ciênc Saúde Coletiva. 2013;18(12):3483-93.

22. Caetano AS, Tavares MCGCF, Lopes MHBM. Incontinência urinária e a prática de atividades físicas. Rev Bras Med Esporte. 2007;13(4):270-4.

23. Nusbaum ML, Gordon M, Nusbaum D, McCarthy MA, Vasilakis D. Smoke alarm: a review of the clinical impact of smoking on women. Prim Care Update $\mathrm{Ob}$ Gyns. 2000;7(5):207-4.

24. Figueiredo EM, Lara JO, Cruz MC, Quintão DMG, Monteiro MCV. Perfil sociodemográfico e clínico de usuárias de Serviço de Fisioterapia Uroginecológica da rede pública. Rev Bras Fisioter. 2008;12(2):136-42.

25. Oliveira E, Zuliani LMM, Ishicava J, Silva SV, Albuquerque SSR, Souza AMB, Barbosa CP. Avaliação dos fatores relacionados à ocorrência da incontinência urinária feminina. Rev Assoc Med Bras. 2010;56(6):688-90.

26. Carvalho CCM, Souza ASR, Moraes Filho OB. Episiotomia seletiva: avanços baseados em evidências. Femina. 2010;38(5):265-70.

27. Doshi AM, Van den Eeden SK, Morrill MY, Schembri M, Thom DH, Brown JS. Women with diabetes: understanding urinary incontinence and help seeking behavior. J Urol. 2010;184(4):1402-7.

28. Danforth KN, Townsend MK, Curhan GC, Resnick NM, Grodstein F. Type 2 diabetes mellitus and risk of stress, urge, and mixed urinary incontinence. J Urol. 2009; 181(1):193-7.

29. Waetjen EL, Feng WY, Ye J, Johnson WO, Greendale GA, Sampselle CM, et al. Factors Associated with worsening and improving urinary incontinence across the menopausal transition. Obstet Gynecol. 2008;111(3):667-77.

30. Albuquerque MT, Micussi BC, Soares EMM, Lemos TMAM, Brito TNS, Silva JB, et al. Correlation between complaints of stress urinary incontinence and the one-hour pad test in postmenopausal women. Rev Bras Ginecol Obstet. 2011;33(2):70-4.

31. Monteiro MVC, Fonseca AMR, Silva Filho AL. Valor do estudo urodinâmico no tratamento da incontinência urinária. Femina. 2012;40(3):135-9.

\section{Endereço para correspondência:}

Karla Veruska Marques Cavalcante

Universidade Federal da Paraíba

Jardim Universitário, s/n - Campus I

CEP: 58051-900 - João Pessoa - PB - Brasil

E-mail: karlaveruska@yahoo.com.br 